\title{
Comparative transcriptomics characterized the distinct biosynthetic abilities of terpenoid and paeoniflorin biosynthesis in herbaceous peony strains
}

\author{
Baowei Lu ${ }^{1}$, Fengxia An ${ }^{\text {Corresp., }}{ }^{\text {, Liangjing Cao }}{ }^{2}$, Qian Gao ${ }^{2}$, Xuan Wang ${ }^{3}$, Yongjian Yang ${ }^{1}$, Pengming Liu ${ }^{1}$, \\ Baoliang Yang ${ }^{1}$, Tong Chen ${ }^{4}$, Xin-Chang Li ${ }^{2}$, Qinghua Chen ${ }^{1}$, Jun Liu ${ }^{\text {Corresp. } 2}$ \\ ${ }^{1}$ Bozhou University, Bozhou, Anhui, China \\ 2 National Key Facility for Crop Resources and Genetic Improvement, Institute of Crop Science, Chinese Academy of Agricultural Sciences, Beijing, China \\ 3 Department of Biological Sciences, Xian Jiaotong-Liverpool University, Suzhou, China \\ 4 National Resource Center for Chinese Materia Medica, Academy of Chinese Medical Sciences, Beijing, China \\ Corresponding Authors: Fengxia An, Jun Liu \\ Email address: An_fengxia@163.com, liujun@caas.cn
}

The herbaceous peony (Paeonia lactiflora Pall.) is a perennial flowering plant of the Paeoniaceae species that is widely cultivated for medical and ornamental uses. The monoterpene glucoside paeoniflorin and its derivatives are the active compounds of the P.lactiflora roots. However, the gene regulation pathways associated with monoterpene and paeoniflorin biosynthesis in P.lactiflora are still unclear. Here, we selected three genotypes of $P$.lactiflora with distinct morphologic features and chemical compositions that were a result of long-term reproductive isolation. We performed an RNA-sequencing experiment to profile the transcriptome changes of the shoots and roots. Using de novo assembly analysis, we identified 36,264 unigenes, including 521 genes responsible for encoding transcription factors. We also identified 28,925 unigenes that were differentially expressed in different organs and/or genotypes. Pathway enrichment analysis showed that the P.lactiflora unigenes were significantly overrepresented in several secondary metabolite biosynthesis pathways. We identified and profiled 33 genes responsible for encoding the enzymescontrolling the major catalytic reactions in the terpenoid backbone and in monoterpenoid biosynthesis. Our study identified the candidate genes in the terpenoid biosynthesis pathways, providing useful information for metabolic engineering of P.lactiflora intended for pharmaceutical uses and facilitating the development of strategies to improve marker-assist P.lactiflora in the future. 


\section{Comparative Transcriptomics Characterized the Distinct}

\section{Biosynthetic Abilities of Terpenoid and Paeoniflorin Biosynthesis in}

\section{Herbaceous Peony Strains}

4 Baowei $\mathrm{Lu}^{1}$, Fengxia $\mathrm{An}^{1 *}$, Liang-Jing $\mathrm{Cao}^{2}$, Qian $\mathrm{Gao}^{2}$, Xuan Wang ${ }^{3}$, Yongjian Yang

5 Pengming Liu ${ }^{1}$, Baoliang Yang ${ }^{1}$, Tong Chen ${ }^{4}$, Xin-Chang Li ${ }^{2}$, Qinghua Chen ${ }^{1}$ and Jun Liu ${ }^{2 *}$

7 'Bozhou University, Bozhou, Anhui, China

$8{ }^{2}$ National Key Facility for Crop Resources and Genetic Improvement, Institute of Crop Science, Chinese

9 Academy of Agricultural Sciences, Beijing 100081, China

${ }^{3}$ Department of Biological Sciences, Xian Jiaotong-Liverpool University, Suzhou 215123, China

${ }^{4}$ National Resource Center for Chinese Materia Medica, Academy of Chinese Medical Sciences, Beijing 100700, China

Corresponding Author:

FengxiaAn (An_fengxia@163.com) or Jun Liu (liujun@caas.cn) 


\section{Abstract}

33 The herbaceous peony (Paeonia lactiflora Pall.) is a perennial flowering plant of the Paeoniaceae species that is 34 widely cultivated for medical and ornamental uses. The monoterpene glucoside paeoniflorin and its derivatives are 35 the active compounds of the P.lactiflora roots. However, the gene regulation pathways associated with monoterpene and paeoniflorin biosynthesis in P. lactiflora are still unclear. Here, we selected three genotypes of P. lactiflora with distinct morphologic features and chemical compositions that were a result of long-term reproductive isolation. We performed an RNA-sequencing experiment to profile the transcriptome changes of the shoots and roots. Using de novo assembly analysis, we identified 36,264 unigenes, including 521 genes responsible for encoding transcription factors. We also identified 28,925 unigenes that were differentially expressed among organs and/or genotypes. Pathway enrichment analysis showed that the P. lactiflora unigenes were significantly overrepresented in several secondary metabolite biosynthesis pathways. We identified and profiled 33 genes responsible for encoding the enzymes controlling the major catalytic reactions in the terpenoid backbone and in monoterpenoid biosynthesis. Our study identified the candidate genes in the terpenoid biosynthesis pathways, providing useful information for metabolic engineering of $P$. lactiflora intended for pharmaceutical uses and facilitating the development of strategies to improve marker-assist $P$. lactiflora breeding in the future. 


\section{Introduction}

60 The herbaceous peony (Paeonialactiflora Pall.) is a flowering plant in the family Paeoniaceae, which is native to Central and eastern Asia (Zhao et al. 2018; Zhao et al. 2017). Its dried root is harvested without the bark in the autumn from plants that are between 3-5 years of age; this harvested material is named Radix Paeoniae Alba or Baishao and is a well-known Chinese herb, used for over 2000 years (He \& Dai 2011; Zha et al. 2012). A water/ethanol extract of Radix Paeoniae Alba, now known as Total Glucosides of Peony (TGP), was originally used in the treatment of typhoid fever (Li et al. 2011). Subsequently, TGP has been widely prescribed for fever, rheumatoid arthritis, hepatitis, muscle cramping and spasms, systemic lupus erythematosus, and dysmenorrhea (Fan et al. 2012; He \& Dai 2011; Ji et al. 2013; Mao et al. 2012; Nam et al. 2013). roots. In vitro and in vivo studies in animal models have confirmed that TGP, paeoniflorin, benzoylpaeoniflorin, galloylpaeoniflorin and their derivatives, are medicinally active compounds with multiple pharmacological effects (Fan et al. 2012; He \& Dai 2011; Zhou \& Wink 2018). TGP can inhibit acute and subacute inflammation, an effect which is potentially mediated by the suppression of prostaglandin E2, leukotriene B4, and nitric oxide, as well as the intracellular calcium ion concentration (He \& Dai 2011; Xu et al. 2016). TGP has been known to protect cells against $\mathrm{Ca}^{2+}$ overload and oxidative stress (Zhang et al. 2017). Moreover, the components of TGP, as important immunomodulatory effectors, can regulate the proliferation and apoptosis of lymphocytes and balance the production of proinflammatory cytokines in a dose-dependent manner (He \& Dai 2011; Hu et al. 2018). In addition, paeoniflorin and its derivatives were shown to inhibit tumor growth and macrophage-mediated lung metastases (Ou et al. 2011; Wu et al. 2015).

Paeoniflorin is a monoterpene glucoside that is biosynthesized from geranyl-pyrophosphate (GPP). GPP is produced via a conversion from the universal terpenoid precursor, Isopentenyl pyrophosphate (IPP). In plants and bacteria, IPP is produced from the two terpene biosynthesis pathways, the mevalonate pathway 
84

85

86

87

88

89

90

91

92

93

(Fig.1) (Kanehisa et al. 2012; Ren et al. 2009; Xie et al. 2011). The MVA pathway reactions take place in the cytosol and are catalyzed by enzymes including hydroxyl methylglutaryl-CoA synthase (HMGCS), acetylCoA C-acetyltransferase (AACT), HMG-CoA reductase (HMGCR), mevalonate kinase (MK), and phosphomevalonate kinase (PMK). The DXP/MEP pathway is catalyzed in the plastids. Pyruvate and glyceraldehyde 3-phosphate are converted by 1-deoxy-D-xylulose-5-phosphate synthase(DXS) and 1-deoxy-Dxylulose-5-phosphate reductoisomerase(DXR) to 1-deoxy-D-xylulose 5-phosphate and 2-C-methyl-Derythritol 4-phosphate, respectively. The products are subsequently catalyzed by 4-diphosphocytidyl-2-Cmethyl-D-erythritol synthase (ISPD), CDP-ME kinase (CDPME), and 2-C-methyl-D-erythritol2,4cyclodiphosphate synthase (ISPF) to mediate the formation of 2-C-methyl-D-erythritol 2,4cyclopyrophosphate, which is then converted to (E)-4-hydroxy-3-methyl-but-2-enyl pyrophosphate (HMB-PP) by HMB-PP synthase (HDS). HMB-PP is converted to IPP and dimethylallyl pyrophosphate (DMAPP) by HMB-PP reductase (ISPH). IPP and DMAPP are condensed by geranyl pyrophosphate synthase(GPS) to produce GPP. In addition to producing a monoterpene, GPP is also a precursor to sesquiterpenes and diterpenes. The conversion from GPP to alpha-terpineol is critical for producing the monoterpene, which is catalyzed by (-)-alpha-terpineolsynthase (RLC1). Paeoniflorin can be modified by benzoic acid and gallic acid to produce benzoylpaeoniflorin and galloylpaeoniflorin, respectively. Benzoic acid and gallic acid are catalyzed by 3-deoxy-7-phosphoheptulonate synthase, 3-dehydroquinate synthase, and 3dehydroquinatedehydratase/shikimate dehydrogenase.

With a long history of domestication and selection, the $P$. lactiflora strains used for medical purposes contain high levels of paeoniflorin and are nearly completely infertile due to embryo abortion in their traditional planting regions, like the Bozhou area. Strains have been reproduced through the vegetative propagation of shoots for hundreds of years. Thus these P. lactiflora accessions are reproductively isolated and may serve as suitable resources in the investigation of the genetic and molecular basis of the paeoniflorin biosynthesis pathways. Using sequence homology to search for the known sequences and domains, several studies identified a group of genes involved in paeoniflorin biosynthesis. For example, a previous study identified 24 genes, including 8 with full-length cDNA sequences and revealed transcriptional and phylogenetic associations with paeoniflorin biosynthesis (Fig.1) (Yuan et al. 2013). However, the genes in the 
111 paeoniflorin biosynthesis pathways and their expression patterns have not been profiled in P. lactiflora strains 112 on a genome-wide basis.

113 High throughput sequencing technologies have revolutionized genomic and transcriptomic studies. 114 Improved algorithms are now available for de novo reassembly of the transcriptome of a non-model plant

115

116 species without a valid reference genome sequence (Luo et al. 2017b). In this study, we assembled the transcriptome of roots and shoots derived from the 3 strains of P. lactiflora using de novo assembly analysis. By aligning the assembled genes with public databases, we globally annotated 34,203 unigenes in $P$. lactiflora. For instance, our analysis identified 521 transcription factor genes. Moreover, we profiled gene expression levels and identified a group of tissue- and/or strain-differential expressed genes using differential expression analysis. From the list of differentially expressed genes among shoots vs. roots, we used homology based annotation with previously known gene families to identify the genes not yet identified from the known pathway.We verified the expression pattern of a selective group of candidate genes using the qRT-PCR assay. Our study provides a valuable dataset for updating our understanding of the gene regulatory network underlying paeoniflorin biosynthesis in P. lactiflora.

\section{Materials and Methods}

\section{Plant materials for RNA-Seq and HPLC}

The P. lactiflora Pu-Bang, Xian-Tiao, and Guan-Shang strains were conserved and cultivated under field conditions at Bozhou University, Bozhou, China. The shoots and roots of 3-year old plants were isolated. To avoid circadian effects, we harvested all the tissues in the afternoon of the same day. The samples for RNAseq with three biological replicates were frozen in liquid nitrogen immediately after harvesting. The isolated samples and purified RNA were stored at $-80{ }^{\circ} \mathrm{C}$. To measure paeoniflorin level by HPLC, roots of 3-year old plants were dried after removing its barks.

\section{RNA extraction, library construction, and Illumina sequencing}

The total RNA of individual samples was extracted and purified with the RNeasy ${ }^{\circledR}$ Plant Mini Kit (QIAGEN, Germany). RNA concentration was measured using a Nanodrop 2100 spectrophotometer. RNA Integrity values were checked using an Agilent Bioanalyzer. The samples with a RIN score $>8.5$ were used for

Peer) reviewing PDF | (2019:03:35941:3:1:NEW 21 Feb 2020) 
138 library construction (Liu et al. 2014). The sequencing libraries were generated using a NEB Next Ultra RNA

139 Library Prep Kit for Illumina (New England Biosystems), following the manufacturer's recommendations.

140 Library sequencing was performed on a Hiseq X10 system with 150-cycle paired-end sequencing protocol

141 (Illumina).

\section{Bioinformatics analysis of RNA-seq datasets}

143 RNA-seq datasets were checked using FastQC (Brown et al. 2017). Referring the methods used in recent

144 studies with modification (Bedre et al. 2016; Lu et al. 2018), we assembled the transcriptome using Trinity

145 (Haas et al. 2013). We aligned read sequences using HISAT2 (Kim et al. 2015). Read count of each gene was

146 called using HTseq-count (Anders et al. 2015).Fragments per kilobase of exon per million fragments mapped

147 of assembled transcripts (FPKM) were calculated and normalized using DESeq2 with global normalization

148 parameters (Anders et al. 2014; Love et al. 2014; Quinn \& Chang 2016; Zhang et al. 2014). The sequences of

149 the assembled unigenes were annotated by Trinotate (Haas et al. 2013). Coding regions of unigenes were 150 predicted using Transdecoder (Haas et al. 2013). BLAST v2.7.1 was performed to determine the sequence 151 homology (e-value cutoff of $1 \mathrm{e}^{-5}$ ) to UniProt/Swiss-Prot, HMMR v3.1b2, EggNOG v4.5.1, and metabolic 152 pathways were analyzed using KEGG database (Kanehisa et al. 2015). For the unigene with annotated 153 homolog, we used the criterion of FPKM more than 0.8 to filter out the lowly expressed genes; for those 154 without homolog, we used FPKM more than 1 as the cut-off criterion. Differential expression analysis was 155 carried out using DESeq2 (Anders \& Huber 2010). Genes with normalized fold-change greater than 2, 156 significance $P$-value less than 0.05 , and Benjamini-Hochberg false discovery rate less than 0.1 were considered 157 to be differentially expressed genes.

\section{Quantitative detection of paeoniflorin}

We referred to the previous method in order to measure paeoniflorin in samples (Yuan et al. 2013). The dried samples $(0.50 \mathrm{~g})$ were weighed and extracted with $50 \mathrm{~mL}$ of $50 \%$ aqueous methanol with ultrasonication

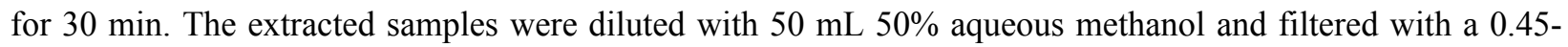
$\mu \mathrm{m}$ Millipore filter membrane (Millipore, MA, USA) at $25^{\circ} \mathrm{C}$. We used the Agilent $1200 \mathrm{LC}$ Series (Agilent Technologies, Palo Alto, CA, USA) High Performance Liquid Chromatography (HPLC) system to measure the paeoniflorin abundance. The wavelength was set at $230 \mathrm{~nm}$ with a flow rate of $1.0 \mathrm{~mL} / \mathrm{min}$ at a temperature of 
166 linearity of the standard compounds was checked at seven concentration solutions.

\section{Quantitative RT-PCR}

A total of 1 to $2 \mu \mathrm{g}$ RNA samples were treated by DNase I (RNeasy plant mini kit) and were reverse transcribed with oligo (dT) primer and SuperScript III (Invitrogen). cDNA samples were analyzed using quantitative PCR with SYBR Premix Ex Taq (Takara) and a Biorad CFX96 real-time PCR system. The P. lactiflora Actin transcript sequence (Available at https://www.ncbi.nlm.nih.gov/nuccore/JN105299) was used as endogenous reference genes to design primers to normalize the expression levels among samples (Qi et al. 2018; Yuan et al. 2013). The qRT-PCR reactions were carried out with two-step cycles $\left(5\right.$ second $95^{\circ} \mathrm{C}$ denaturation, 30 second $60^{\circ} \mathrm{C}$ annealing and extension) and 45 cycles of amplification (Supplemental Fig. 1A). The melting curves of primers were checked to ensure the primer efficiencies (Supplemental Fig. 1B-L). We used three technical replicates to produce the average expression levels of the genes relative to that of the reference gene using the $2^{-\Delta \Delta C T}$ method (Livak \& Schmittgen 2001). The primers are listed in Supplemental Table 1.

\section{Results}

\section{RNA-Seq and de novo assembly of the P. lactiflora transcriptome}

The P. lactiflora Pu-Bang (PB) and Xian-Tiao (XT) accessions are the most widely used herbaceous stains for medical uses due to their high levels of paeoniflorin, which is derived mainly from roots of 3-year old plants; whereas the Guan-Shang (GS) accession contains less paeoniflorin and is usually cultivated for ornamental uses. biosynthesis in P. lactiflora, we purified the RNA samples with three biological replicates derived from the shoots and roots of 3-year old PB, XT, and GS plants, and carried out the RNA-sequencing analysis using the 
193

194

reads in total (Supplemental table 2). Using Trinity (Haas et al. 2013), we performed de novo transcriptome assembly and obtained 36,264 unigenes encoding 72,910 transcripts with $986 \mathrm{nt}$ contig N50 length and 42.7\% average GC content (Table 1). We also checked the completeness of de novo assembled Unigenes by aligning the sequences with Swiss-Prot database (TheUniProtConsortium. 2017). The results showed that 7,131 (50\%) out of the 14,165 aligned sequences have more than $40 \%$ coverage of known transcripts (Supplemental table 3 $\& 4)$.

\section{Functional annotation of expressed genes in the three $P$. lactiflora accessions}

Due to embryo abortion and vegetative propagation, the $P$. lactiflora accessions have been undergoing reproductive isolation with a long cultivation history in the Bozhou region and were thought to be genetically distinct from each other (Zhou et al. 2002). However, the genomic evidence supporting this point is still lacking. We measured the expression levels of unigenes by calculating normalized Fragments Per Kilobase of exon per million fragments Mapped (FPKM). The unigenes with an FPKM value higher than 1 in at least one were used to perform hierarchical clustering analysis based on the Pearson correlation efficiency. We analyzed the hierarchical structure of the gene expression levels on a genome-wide basis (Supplemental Fig. 2A). Most of the biological replicate samples belonged to the same clusters. Principal component analysis results also showed a similar result confirming a high level of reproducibility of the biological replicate samples (Supplemental Fig. 2B). However, the tissues and strains were distributed in different cluster clades. It was noted that all the clades derived from PB and XT were separated from those of GS, indicating that the strains for medicinal uses are genetically divergent from the strains used for ornamental purposes, possibly due to the selection and reproductive isolation among the strains.

We predicted the protein-coding potential for the unigenes using the Transdecoder and searched for the annotation for the unigenes by aligning the assembled transcripts and predicted peptide sequences to the protein sequences annotated by Swiss-Prot, TrEMBL, Pfam, and KEGG databases using Trinotate, BLASTP, and BLASTX (Boutet et al. 2016; Camon et al. 2003; El-Gebali et al. 2019; Haas et al. 2013; Kanehisa et al. 2017). In total, we identified 34,203 unigenes containing significant matches to the annotated genes/proteins in at least one database (Fig. 3B). Of them, 28,083 (82\%) were reproducibly detected by at least 2 data resources. The annotation information, predicted protein sequences, and FASTA-formatted sequences of these genes 
220 221 (Supplemental table 3).

222

223

were provided in supplemental materials that could serve as a reference annotation for future studies

Transcription factors (TFs) with DNA-binding domains are the major regulators controlling the activity and specificity of the gene transcription process. We predicted genes encoding TFs in our assembled unigene dataset and identified $521 \mathrm{TF}$-encoding unigenes belonging to $32 \mathrm{TF}$ families (Fig. 3C). Of these, AP2/ERF, bHLH, FAR1, bZIP, and HB are the most abundant TF genes. The detailed information of the TF genes was provided in supplemental Table 5.

\section{Identification of tissue- and/or strain-differentially expressed unigenes}

Next, we searched for the differentially expressed unigenes. For each strain, we compared shoots and roots and extracted lists of root/shoot specific upregulated genes (fold change of expression level $>2$ and false discovery rate $<0.05$ ). Our analysis identified 10,125 up-regulated unigenes in roots and 11,911 in shoots. There were 1906 to 3737 unigenes specifically up-regulated in the roots and/or shoots of each strain; whereas only $332(3 \%)$ and $886(7 \%)$ unigenes were up-regulated in the roots and shoots of all the three P. lactiflora strains, respectively (Fig. 4). This result showed that a large number of the tissue-specific genes were also strain-specific, which is consistent with the fact that the three $P$. lactiflora strains have been undergoing genetic separation and selection during the last several centuries.

We performed the correlation analysis between the paeoniflorin levels and the expression levels of all the genes in shoots and roots of the three strains (supplemental table 3). Our analysis identified 3952 genes with the Pearson correlation coefficients higher than 0.6. Among the 161 gene with Pearson correlation coefficients higher than 0.9, we found several genes encoding transcription factors, E3 ubiquitin-protein ligase and oxidation-reduction related process, such as GT-2, ABCF4, SKP2A, FREE, GOR, TLP, RMA1H1, HAT5, XBAT31 and DELLA, suggesting transcriptional and post-translational regulation may be highly associated with paeoniflorin accumulation.

\section{Identification of genes in terpenoid and paeoniflorin biosynthesis pathway}

To further dissect the regulation pathways of the unigenes, we analyzed the gene list enrichment using the Kyoto Encyclopedia of Genes and Genomes (KEGG) datasets and KOBAS3.0 with hypergeometric testing and Benjamini and Hochberg correction (Kanehisa et al. 2012; Xie et al. 2011). In total, we identified 71 
247 significantly enriched KEGG pathways in P. lactiflora (Supplemental Table 6). The list included several

248 KEGG terms of secondary metabolite biosynthesis, such as: Terpenoid backbone biosynthesis $(P$-value $<$ 249 0.0152), Glycerophospholipid metabolism $(P$-value $<0.0001)$, Inositol phosphate metabolism $(P$-value $<$ 250 0.0001), Pyruvate metabolism $(P$-value $<0.0002)$, Seleno compound metabolism $(P$-value $<0.0298)$, 251 Ascorbate and aldarate metabolism $(P$-value $<0.0172)$ and Butanoate metabolism $(P$-value $<0.0352)$. 252 P. lactiflora are generally known to have abundant secondary metabolites (Li et al. 2016a; Liu et al. 2017; Ma 253 et al. 2016). Our transcriptome and pathway enrichment results are consistent with the metabolism profiling 254 studies.

255 The previous studies have identified 19 EST sequences in the terpenoid backbone biosynthesis pathways in P. lactiflora, including 7 with full-length cDNA sequences (Yuan et al. 2013). However, the genes in the terpenoid backbone biosynthesis pathway have not been globally profiled and the enzyme catalyzing the initiation step from GPP to monoterpenoid biosynthesis has not been identified in P. lactiflora yet. In our datasets, we identified 32 genes with full-length CDSs encoding the enzymes controlling the major catalytic reactions in MVA and MEP pathways (Supplemental Table 7). Compared with the previous study (Yuan et al. 2013), the genes encoding 10 previously reported enzymes were also identified in our study (Fig. 1). Moreover, we identified the unigene (E_H33980_c1_g4, RLC1) encoding (-)-alpha-terpineol synthase (EC 4.2.3.111) that can catalyze the conversion from GPP to alpha-terpineol (Kulkarni et al. 2013b), a monoterpene precursor of paeoniflorin. Our analysis identified the genes encoding the enzymes that almost completely catalyzed the reactions from the glycolysis products to the terpenoid backbone and monoterpenoid biosynthesis in P. lactiflora.

The high accumulation of paeoniflorin in XT and/or BP roots suggests some genes in the paeoniflorin biosynthesis pathway may be highly expressed in XT and/or PB roots. We analyzed the expression patterns of the aforementioned 33 unigenes (Fig 5). In XT roots, the expression levels of $A A C T 1, H M G S, M K, P M K, G G R$, FPS1 were higher than those in GS roots; whereas the expression levels of $P M K, M V D, G G R$ in PB roots were expression patterns. Note that XT and PB presented the distinct expression pattern of these genes, which was 
274 To confirm the expression levels determined by the RNA-seq experiment, we used a quantitative Real275 time PCR (qRT-PCR) assay to measure the expression levels of several paeoniflorin biosynthesis pathway 276 genes and transcription factor genes (Fig. $6 \&$ Supplemental Fig. 1). The expression levels of the transcription 277 factor gene BHLH94 showed a highly correlation with paeoniflorin levels. The paeoniflorin biosynthesis pathway gene FPS1, AACT1, HMGS, IPI2 and $M K$ as well as the transcription factor gene DREBID and NAC098 presented root- and/or strain-preferential expression patterns. Our qRT-PCR verification results were highly consistent with the RNA-seq analyses (Fig. 6 \& Supplemental Fig. 3).

281

\section{Discussion}

The herbaceous peony has a large and complex genome. It was estimated that the genome size of the tree peony, a closely related species of P. lactiflora, is about $12.5 \mathrm{Gbp}$. Our analysis showed that many gene families have multiple copies also suggesting the chromosome duplication and/or gene family expansion event(s) in P. lactiflora genome. Thus, assembling the high-quality genome of $P$. lactiflora remains a challenge. Recently, transcriptome studies on a large number of plant species with complex genomes, such as smooth cordgrass (Spartina alterniflora) (Bedre et al. 2016), buckwheat (Lu et al. 2018), and Caraganakor shinskii (Li et al. 2016b), have been carried out. Transcriptome assembly is currently a feasible and cost-efficient technology to globally identify genes in the P. lactiflora genome because genome information is unavailable (Luo et al. 2017a; Luo et al. 2017b). In our study, we identified 36,264 unigenes. Using the homolog alignment analysis, we identified a large number of these assembled unigenes encoded in known regulatory domains/motifs, suggesting their molecular functions. Of these, we identified 521 transcription factor genes belonging to 32 families and profiled their expression patterns. Our study identified a large number of previously un-annotated genes in the P. lactiflora genome, which could supply valuable molecular information for future functional studies.

Plant terpenoids are widely used as traditional herbal remedies and for their aromatic qualities. Terpenoids are highly abundant in several accessions of $P$. lactiflora, suggesting a high potential for terpenoid biosynthesis in them. However, the terpenoid biosynthetic pathways are not yet fully understood in P. lactiflora. Previous studies have identified the 19 EST sequences in the terpenoid backbone biosynthesis pathway in P. lactiflora (Yuan et al. 2013). Paeoniflorin is accumulating in roots of 3-year-old plants and 
301 lacking in leaves and its accumulation levels are highly differed in different strains (Li et al. 2011; Yuan et al. 302 2013). The tissue- and strain-specific accumulation pattern of paeoniflorin facilitates us to explore the 303 molecular basis of paeoniflorin biosynthesis pathways. In our study, we carry out a transcriptome analysis and 304 identify 32 unigenes in the pathway and profiled their expression patterns that covered most of the reactions in 305 the terpenoid backbone biosynthesis pathway. The expression levels of AACT1 showed specific accumulation in XT/PB roots; whereas most of the genes in the terpene backbone biosynthesis showed differential expression patterns between strains rather between tissues. This result raised two testable hypotheses: 1) The several genes with $A A C T 1$ expression patterns are master genes in the pathway; 2) The root-specific expression patterns of these genes associated with paeoniflorin levels could be found in other strains. The gene sequences and their expression patterns identified in our study could serve as a reference dataset for follow up studies to test their expression specificities in more P. lactiflora strains associated with paeoniflorin levels. With this data, the other unidentified gene members and the complete terpenoid backbone biosynthesis pathway in P. lactiflora strains can be deciphered in the future, using homologous cloning, phylogenetic analysis, and catalytic kinetics experiments.

Our transcriptome profiling analysis uncovered the differential expression patterns of roots vs. shoots of three P. lactiflora. strains, with different paeoniflorin contents in the roots, which is the producing tissue and had been determined higher in two strains. Our results suggested the genes in the terpenoid biosynthesis pathway were diversified during the selection process, which could supply valuable molecular information for future functional studies in P. lactiflora. Geranyldiphosphate synthases and farnesyldiphosphate synthase catalyze the important branch-point reactions from GPP to monoterpenes and sesquiterpenes. The catalytic activities of the two enzymes are sensitive to temperature and metal ion concentration (Kulkarni et al. 2013a). Under high temperature and $\mathrm{Mg}^{2+}$ rich condition, the monoterpene concentration was significant higher compared with those of sesquiterpenes. In our experiment, we identified several unigenes encoding Geranyldiphosphate synthases and farnesyldiphosphate synthase in P. lactiflora. Their catalytic activities could be investigated under different temperature and metal ion conditions in follow-up studies. 
It has been known that $P$. lactiflora is highly sensitive to the photoperiod and temperature changes. The accessions grown in the Bozhou region have been undergoing reproductive isolation for a long period. Thus, the genetic background of each individual might be fixed. In our study, we identified a large number of genes in $P$. lactiflora and found gene expression patterns in the terpenoid pathway are highly diversified among different accessions. We found The different biosynthetic abilities of terpenoid biosynthesis among the strains may be largely contributed to by several master genes, suggesting that the heterosis in crosses of PB and XT should be considered as a potential approach to breed $P$. lactiflora strains with high paeoniflorin levels. With the sequences of these genes, the phylogenetic structures and population genetics backgrounds of germplasm resources could be further investigated to elucidate the domestication and selection process of $P$. lactiflora.

\section{Conclusion}

Using the RNA-sequencing protocol, we assembled the exon structures of 36,264 unigenes in the shoots and roots of three 3-year-old P. lactiflorais accessions, including 28,925 differentially expressed genes. We systematically annotated their molecular functions by aligning their sequences with those from multiple data resources. We found 3,952 genes with the Pearson Correlation Coefficients higher than 0.6 between the expression levels and the paeoniflorin levels suggesting their putative functions associated with paeoniflorin biosynthesis. We identified 32 genes encoding the enzymes controlling the major catalytic reactions in MVA and MEP pathways and one gene encoding (-)-alpha-terpineol synthase. These genes contributed nearly complete terpenoid backbone biosynthesis pathways. By profiling gene expression patterns associated with MVP, MEP pathways, and I-PP to monoterpene conversation reactions, we uncovered $A A C T 1, H M G S$, $M K$, $P M K, G G R, F P S 1$ and $M V D$ that were highly expressed in the roots of high-paeoniflorin accessions, indicating that the different biosynthetic abilities of terpenoid biosynthesis among the accessions may be largely contributed to by several master genes.

\section{Author Contributions}

F. A., B. L., and J. L. designed the experiment. B. L., Y. Y., P. L., Y. Z., Q. C. and B. Y. sampled the biological materials and extracted the RNA and sequenced the libraries. T. C., F. A., J.L. and Y. W. analyzed the datasets. B.L. J.L. and Y.W. prepared the figures and wrote the manuscript. All authors reviewed and approved the final version of the manuscript.

PeerJ reviewing PDF | (2019:03:35941:3:1:NEW 21 Feb 2020) 
357

358

359

360

361

362

363

364

365

366

367

368

369

370

371

372

373

374

375

376

377

378

379

380

381

382

383

384

385

386

387

388

389

390

391

392

393

\section{Accession numbers}

The fastq-formatted RNA-seq datasets and the sampling information (accession number CRA001881, https://bigd.big.ac.cn/gsa/browse/CRA001881) are publically available on the Genome Sequence Archive database (BIG.Members. 2018; Wang et al. 2017).

\section{REFERENCE}

Anders S, and Huber W. 2010. Differential expression analysis for sequence count data. Genome biology 11:R106. 10.1186/gb-2010-11-10-r106

Anders S, Pyl PT, and Huber W. 2014. HTSeq-a Python framework to work with high-throughput sequencing data. Bioinformatics:btu638.

Anders S, Pyl PT, and Huber W. 2015. HTSeq--a Python framework to work with high-throughput sequencing data. Bioinformatics 31:166-169. 10.1093/bioinformatics/btu638

Bedre R, Mangu VR, Srivastava S, Sanchez LE, and Baisakh N. 2016. Transcriptome analysis of smooth cordgrass (Spartina alterniflora Loisel), a monocot halophyte, reveals candidate genes involved in its adaptation to salinity. BMC genomics 17:657-657. 10.1186/s12864-016-3017-3

BIG.Members. 2018. Database Resources of the BIG Data Center in 2018. Nucleic acids research 46:D14-D20. 10.1093/nar/gkx897

Boutet E, Lieberherr D, Tognolli M, Schneider M, Bansal P, Bridge AJ, Poux S, Bougueleret L, and Xenarios I. 2016. UniProtKB/Swiss-Prot, the Manually Annotated Section of the UniProt KnowledgeBase: How to Use the Entry View. In: Edwards D, ed. Plant Bioinformatics: Methods and Protocols. New York, NY: Springer New York, 23-54.

Brown J, Pirrung M, and McCue LA. 2017. FQC Dashboard: integrates FastQC results into a web-based, interactive, and extensible FASTQ quality control tool. Bioinformatics. 10.1093/bioinformatics/btx373

Camon E, Barrell D, Brooksbank C, Magrane M, and Apweiler R. 2003. The Gene Ontology Annotation (GOA) Project--Application of GO in SWISS-PROT, TrEMBL and InterPro. Comparative and functional genomics 4:71-74. 10.1002/cfg.235

El-Gebali S, Mistry J, Bateman A, Eddy SR, Luciani A, Potter SC, Qureshi M, Richardson L, Salazar GA, Smart A, Sonnhammer ELL, Hirsh L, Paladin L, Piovesan D, Tosatto SCE, and Finn RD. 2019. The Pfam protein families database in 2019. Nucleic Acids Research 47:D427-D432. 10.1093/nar/gky995

Fan YF, Xie Y, Liu L, Ho HM, Wong YF, Liu ZQ, and Zhou H. 2012. Paeoniflorin reduced acute toxicity of aconitine in rats is associated with the pharmacokinetic alteration of aconitine. Journal of ethnopharmacology 141:701-708. 10.1016/j.jep.2011.09.005

Haas BJ, Papanicolaou A, Yassour M, Grabherr M, Blood PD, Bowden J, Couger MB, Eccles D, Li B, Lieber M, MacManes MD, Ott M, Orvis J, Pochet N, Strozzi F, Weeks N, Westerman R, William T, Dewey CN, Henschel R, LeDuc RD, Friedman N, and Regev A. 2013. De novo transcript sequence reconstruction from RNA-seq using the Trinity platform for reference generation and analysis. Nature protocols 8:1494-1512. $10.1038 /$ nprot.2013.084 
394

395

396

397

398

399

400

401

402

403

404

405

406

407

408

409

410

411

412

413

414

415

416

417

418

419

420

421

422

423

424

425

426

427

428

429

430

431

432

433

434

He DY, and Dai SM. 2011. Anti-inflammatory and immunomodulatory effects of paeonia lactiflora pall., a traditional chinese herbal medicine. Frontiers in pharmacology 2:10. 10.3389/fphar.2011.00010

Hu B, Xu G, Zhang X, Xu L, Zhou H, Ma Z, Shen X, Zhu J, and Shen R. 2018. Paeoniflorin Attenuates Inflammatory Pain by Inhibiting Microglial Activation and Akt-NF-kappaB Signaling in the Central Nervous System. Cellular physiology and biochemistry : international journal of experimental cellular physiology, biochemistry, and pharmacology 47:842-850. 10.1159/000490076

Ji Y, Wang T, Wei ZF, Lu GX, Jiang SD, Xia YF, and Dai Y. 2013. Paeoniflorin, the main active constituent of Paeonia lactiflora roots, attenuates bleomycin-induced pulmonary fibrosis in mice by suppressing the synthesis of type I collagen. Journal of ethnopharmacology 149:825-832. 10.1016/j.jep.2013.08.017

Kanehisa M, Furumichi M, Tanabe M, Sato $Y$, and Morishima K. 2017. KEGG: new perspectives on genomes, pathways, diseases and drugs. Nucleic Acids Research 45:D353-D361. 10.1093/nar/gkw1092

Kanehisa M, Goto S, Sato Y, Furumichi M, and Tanabe M. 2012. KEGG for integration and interpretation of largescale molecular data sets. Nucleic acids research 40:D109-114. 10.1093/nar/gkr988

Kanehisa M, Sato Y, Kawashima M, Furumichi M, and Tanabe M. 2015. KEGG as a reference resource for gene and protein annotation. Nucleic acids research 44:D457-D462.

Kim D, Langmead B, and Salzberg SL. 2015. HISAT: a fast spliced aligner with low memory requirements. Nat Methods 12:357-360. 10.1038/nmeth.3317

nmeth.3317 [pii]

Kulkarni R, Pandit S, Chidley H, Nagel R, Schmidt A, Gershenzon J, Pujari K, Giri A, and Gupta V. 2013a. Characterization of three novel isoprenyl diphosphate synthases from the terpenoid rich mango fruit. Plant physiology and biochemistry : PPB 71:121-131. 10.1016/j.plaphy.2013.07.006

Kulkarni R, Pandit S, Chidley H, Nagel R, Schmidt A, Gershenzon J, Pujari K, Giri A, and Gupta V. 2013b. Characterization of three novel isoprenyl diphosphate synthases from the terpenoid rich mango fruit. Plant Physiology and Biochemistry 71:121-131. https://doi.org/10.1016/j.plaphy.2013.07.006

Li B, Bhandari DR, Rompp A, and Spengler B. 2016a. High-resolution MALDI mass spectrometry imaging of gallotannins and monoterpene glucosides in the root of Paeonia lactiflora. Scientific reports 6:36074. 10.1038/srep36074

Li J, Chen CX, and Shen YH. 2011. Effects of total glucosides from paeony (Paeonia lactiflora Pall) roots on experimental atherosclerosis in rats. Journal of ethnopharmacology 135:469-475. 10.1016/j.jep.2011.03.045

Li S, Fan C, Li Y, Zhang J, Sun J, Chen Y, Tian C, Su X, Lu M, Liang C, and Hu Z. 2016b. Effects of drought and saltstresses on gene expression in Caragana korshinskii seedlings revealed by RNA-seq. BMC genomics 17:200-200. 10.1186/s12864-016-2562-0

Liu D, Wang D, Qin Z, Zhang D, Yin L, Wu L, Colasanti J, Li A, and Mao L. 2014. The SEPALLATA MADS - box protein SLMBP21 forms protein complexes with JOINTLESS and MACROCALYX as a transcription activator for development of the tomato flower abscission zone. The Plant Journal 77:284-296.

Liu P, Xu Y, Yan H, Chen J, Shang E-X, Qian D-W, Jiang S, and Duan J-A. 2017. Characterization of molecular signature of the roots of Paeonia lactiflora during growth. Chinese Journal of Natural Medicines 15:785793. https://doi.org/10.1016/S1875-5364(17)30110-3

Livak KJ, and Schmittgen TD. 2001. Analysis of relative gene expression data using real-time quantitative PCR and the 2- $\Delta \Delta C T$ method. methods 25:402-408.

Peer] reviewing PDF | (2019:03:35941:3:1:NEW 21 Feb 2020) 
435

436

437

438

439

440

441

442

443

444

445

446

447

448

449

450

451

452

453

454

455

456

457

458

459

460

461

462

463

464

465

466

467

468

469

470

471

472

473

474

475

Love MI, Huber W, and Anders S. 2014. Moderated estimation of fold change and dispersion for RNA-seq data with DESeq2. Genome biology 15:550.

Lu Q-H, Wang Y-Q, Song J-N, and Yang H-B. 2018. Transcriptomic identification of salt-related genes and de novo assembly in common buckwheat (F. esculentum). Plant Physiology and Biochemistry 127:299-309. https://doi.org/10.1016/j.plaphy.2018.02.001

Luo J, Duan J, Huo D, Shi Q, Niu L, and Zhang Y. 2017a. Transcriptomic Analysis Reveals Transcription Factors Related to Leaf Anthocyanin Biosynthesis in Paeonia qiui. Molecules 22. 10.3390/molecules22122186

Luo J, Shi Q, Niu L, and Zhang Y. 2017b. Transcriptomic Analysis of Leaf in Tree Peony Reveals Differentially Expressed Pigments Genes. Molecules 22. 10.3390/molecules22020324

Ma X, Chi YH, Niu M, Zhu Y, Zhao YL, Chen Z, Wang JB, Zhang CE, Li JY, Wang LF, Gong M, Wei SZ, Chen C, Zhang L, $\mathrm{Wu} \mathrm{MQ}$, and Xiao XH. 2016. Metabolomics Coupled with Multivariate Data and Pathway Analysis on Potential Biomarkers in Cholestasis and Intervention Effect of Paeonia lactiflora Pall. Frontiers in pharmacology 7:14. 10.3389/fphar.2016.00014

Mao QQ, Ip SP, Xian YF, Hu Z, and Che CT. 2012. Anti-depressant-like effect of peony: a mini-review. Pharmaceutical biology 50:72-77. 10.3109/13880209.2011.602696

Nam KN, Yae CG, Hong JW, Cho DH, Lee JH, and Lee EH. 2013. Paeoniflorin, a monoterpene glycoside, attenuates lipopolysaccharide-induced neuronal injury and brain microglial inflammatory response. Biotechnology letters 35:1183-1189. 10.1007/s10529-013-1192-8

Ou TT, Wu CH, Hsu JD, Chyau CC, Lee HJ, and Wang CJ. 2011. Paeonia lactiflora Pall inhibits bladder cancer growth involving phosphorylation of Chk2 in vitro and in vivo. Journal of ethnopharmacology 135:162-172. 10.1016/j.jep.2011.03.011

Qi Z, Zhang Z, Wang Z, Yu J, Qin H, Mao X, Jiang H, Xin D, Yin Z, Zhu R, Liu C, Yu W, Hu Z, Wu X, Liu J, and Chen Q. 2018. Meta-analysis and transcriptome profiling reveal hub genes for soybean seed storage composition during seed development. Plant, cell \& environment. 10.1111/pce.13175

Quinn JJ, and Chang HY. 2016. Unique features of long non-coding RNA biogenesis and function. Nat Rev Genet 17:47-62. 10.1038/nrg.2015.10

Ren ML, Zhang X, Ding R, Dai Y, Tu FJ, Cheng YY, and Yao XS. 2009. Two new monoterpene glucosides from Paeonia lactiflora Pall. Journal of Asian natural products research 11:670-674. 10.1080/10286020902980087

TheUniProtConsortium. 2017. UniProt: the universal protein knowledgebase. Nucleic acids research 45:D158-D169. 10.1093/nar/gkw1099

Wang Y, Song F, Zhu J, Zhang S, Yang Y, Chen T, Tang B, Dong L, Ding N, Zhang Q, Bai Z, Dong X, Chen H, Sun M, Zhai S, Sun Y, Yu L, Lan L, Xiao J, Fang X, Lei H, Zhang Z, and Zhao W. 2017. GSA: Genome Sequence Archive<sup/>. Genomics, proteomics \& bioinformatics 15:14-18. 10.1016/j.gpb.2017.01.001

Wu Q, Chen GL, Li YJ, Chen Y, and Lin FZ. 2015. Paeoniflorin inhibits macrophage-mediated lung cancer metastasis. Chinese journal of natural medicines 13:925-932. 10.1016/S1875-5364(15)30098-4

Xie C, Mao X, Huang J, Ding Y, Wu J, Dong S, Kong L, Gao G, Li CY, and Wei L. 2011. KOBAS 2.0: a web server for annotation and identification of enriched pathways and diseases. Nucleic acids research 39:W316-322. 10.1093/nar/gkr483

Xu W, Zhao Y, Qin Y, Ge B, Gong W, Wu Y, Li X, Xu P, and Xue M. 2016. Enhancement of Exposure and Reduction of Elimination for Paeoniflorin or Albiflorin via Co-Administration with Total Peony Glucosides and Hypoxic Pharmacokinetics Comparison. Molecules 21. 10.3390/molecules21070874

PeerJ reviewing PDF | (2019:03:35941:3:1:NEW 21 Feb 2020) 
476

477

478

479

480

481

482

483

484

485

486

487

488

489

490

491

492

493

494

495

496

497

498
Yuan Y, Yu J, Jiang C, Li M, Lin S, Wang X, and Huang L. 2013. Functional diversity of genes for the biosynthesis of paeoniflorin and its derivatives in Paeonia. International Journal of Molecular Sciences 14:18502-18519. 10.3390/ijms140918502

Zha LP, Cheng ME, and Peng HS. 2012. Identification of ages and determination of paeoniflorin in roots of Paeonia lactiflora Pall. From four producing areas based on growth rings. Microscopy research and technique 75:1191-1196. 10.1002/jemt.22048

Zhang Y, Qiao L, Xu W, Wang X, Li H, Chu K, and Lin Y. 2017. Paeoniflorin Attenuates Cerebral Ischemia-Induced Injury by Regulating Ca(2+)/CaMKII/CREB Signaling Pathway. Molecules 22. 10.3390/molecules22030359

Zhang YC, Liao JY, Li ZY, Yu Y, Zhang JP, Li QF, Qu LH, Shu WS, and Chen YQ. 2014. Genome-wide screening and functional analysis identify a large number of long noncoding RNAs involved in the sexual reproduction of rice. Genome Biol 15:512. 10.1186/s13059-014-0512-1

Zhao D, Wang R, Liu D, Wu Y, Sun J, and Tao J. 2018. Melatonin and Expression of Tryptophan Decarboxylase Gene (TDC) in Herbaceous Peony (Paeonia lactiflora Pall.) Flowers. Molecules 23. 10.3390/molecules23051164

Zhao D, Wei M, Shi M, Hao Z, and Tao J. 2017. Identification and comparative profiling of miRNAs in herbaceous peony (Paeonia lactiflora Pall.) with red/yellow bicoloured flowers. Scientific reports 7:44926. $10.1038 /$ srep44926

Zhou H, Hu S, Guo B, Feng X, Yan Y, and Li JJYxxbApS. 2002. A study on genetic variation between wild and cultivated populations of Paeonia lactiflora Pall. 37:383-388.

Zhou JX, and Wink M. 2018. Reversal of Multidrug Resistance in Human Colon Cancer and Human Leukemia Cells by Three Plant Extracts and Their Major Secondary Metabolites. Medicines 5. 10.3390/medicines5040123 
Figure 1

The genes encoding enzymes in terpenoid backbone and monoterpenoid biosynthesis pathways in P. lactiflora

The identified $P$. lactiflora enzymes are highlighted by red rectangles. The newly identified enzymes compared with the previous study are indicated by arrows (Yuan et al. 2013).

TERPENOID BACKBONE BIOSYNTHESIS

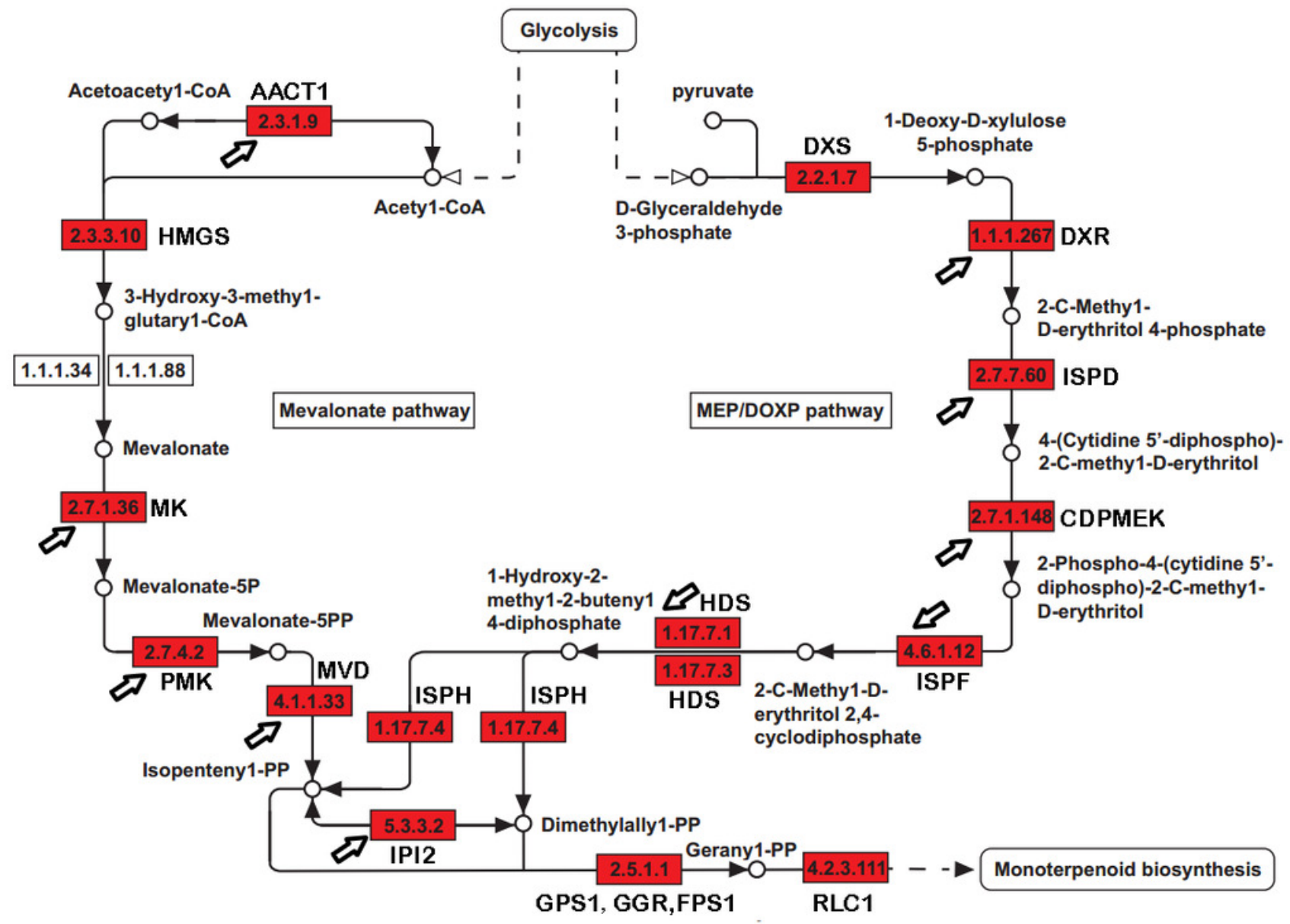




\section{Figure 2}

The developmental features of the three P.lactiflora strains

(A-C) shows the 30-day-old plants of Xian-Tiao (XT), Pu-Bang (PB), and Guan-Shang (GS) strains, which were grown from the shoots (D-F) isolated from 3-year-old plants. (G-I) and (JL) show fresh roots and dried roots without the bark of 3 year-old plants, respectively.

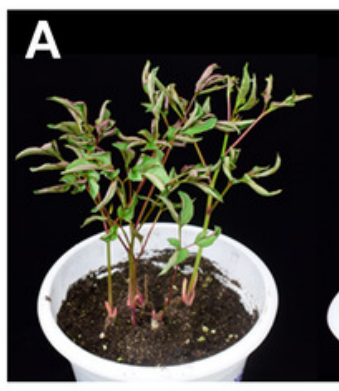

XT

\section{B}

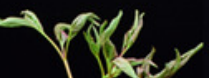

5

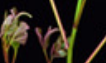

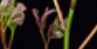

$1 / \%$

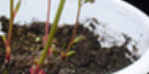

PB

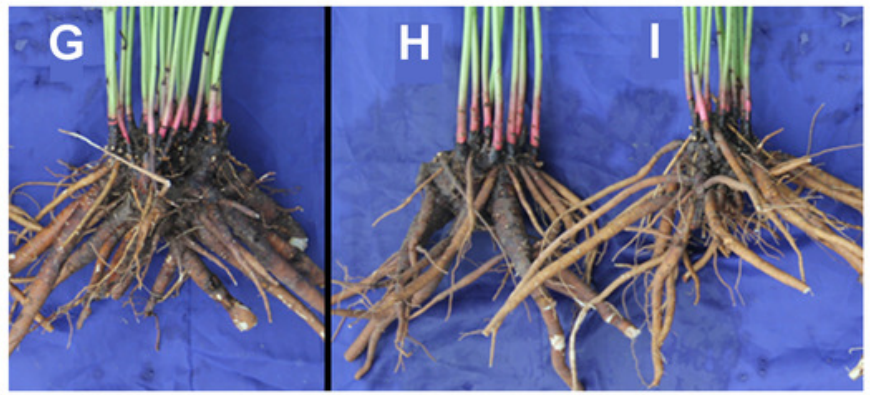

XT

PB

GS

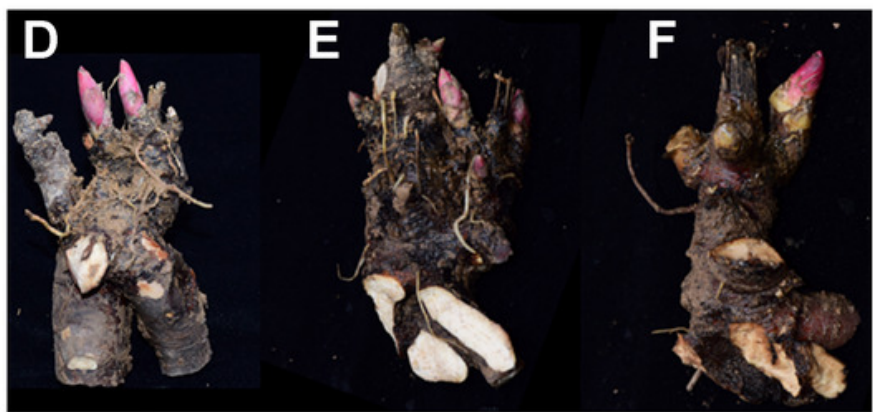

XT

PB

GS

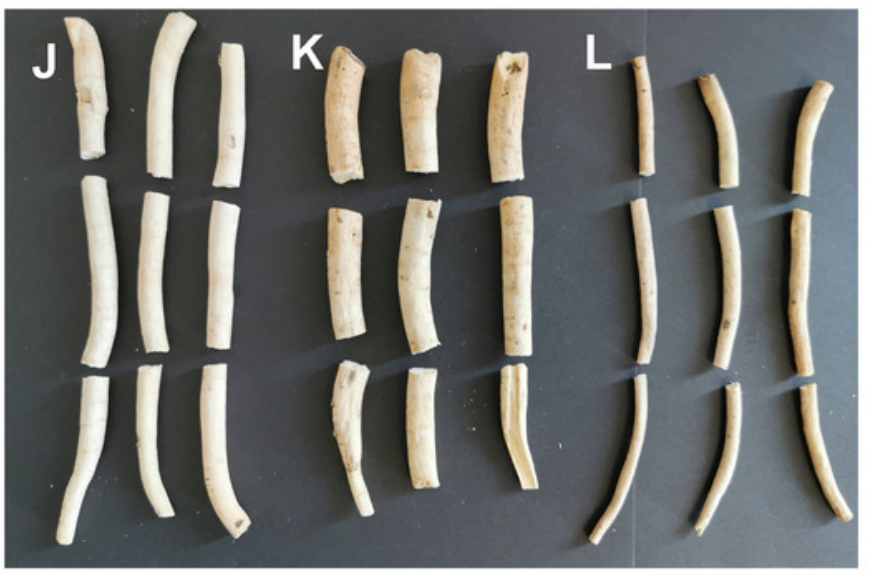

XT
PB

GS 
Figure 3

Paeoniflorin levels and gene annotation in the three P.lactiflora accessions

(A) Paeoniflorin levels measured by HPLC system. Bars show standard deviations of five biological replicates. B) Functional annotation of unigenes using the 4 databases. (C) Transcription factor genes identified in P. lactiflora.
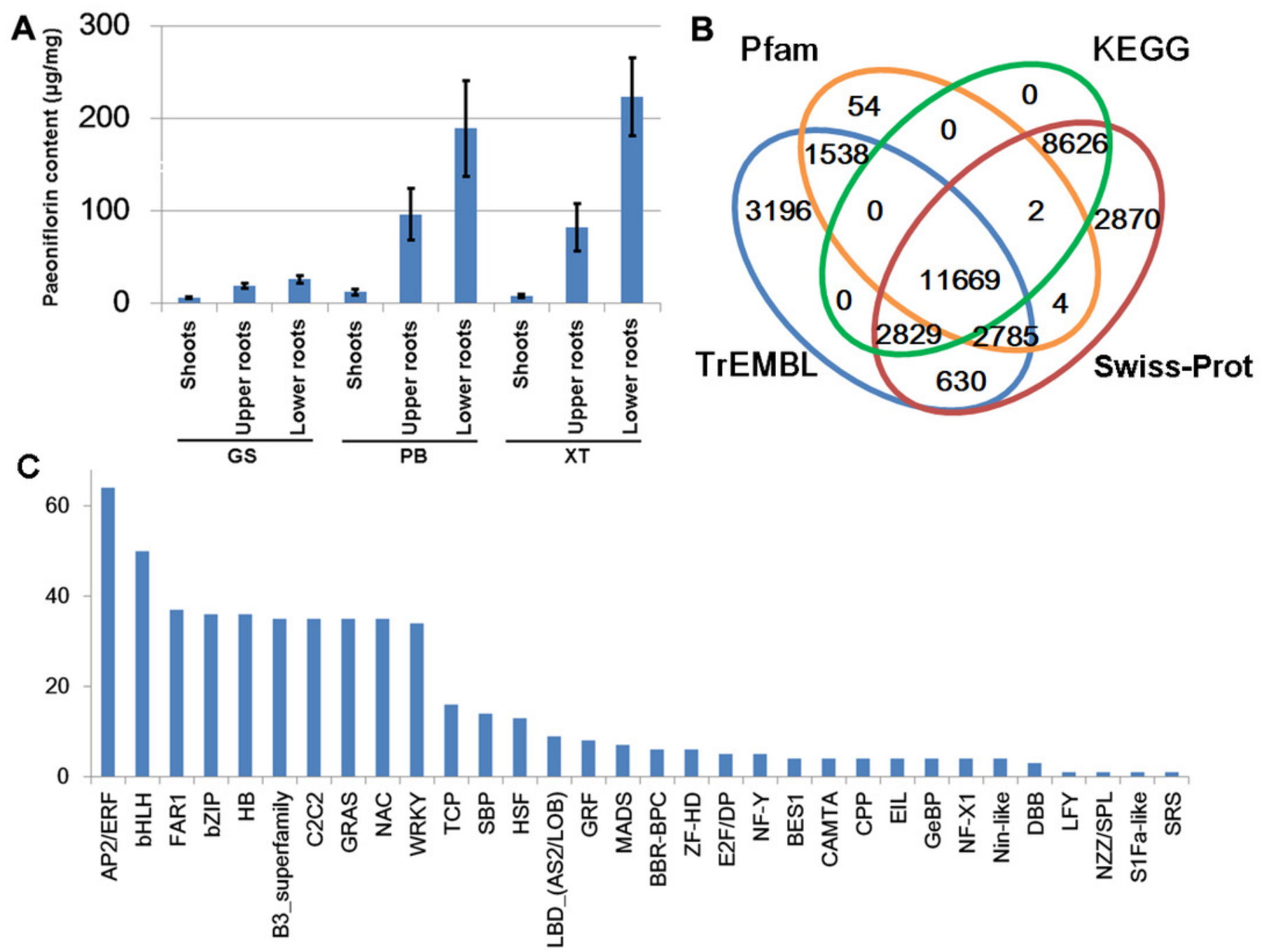
Figure 4

Identification of the differentially expressed genes

The differentially expressed genes in roots $(A)$ and shoots $(B)$ of the three $P$. lactiflora accessions.

A Up-regulated genes in roots

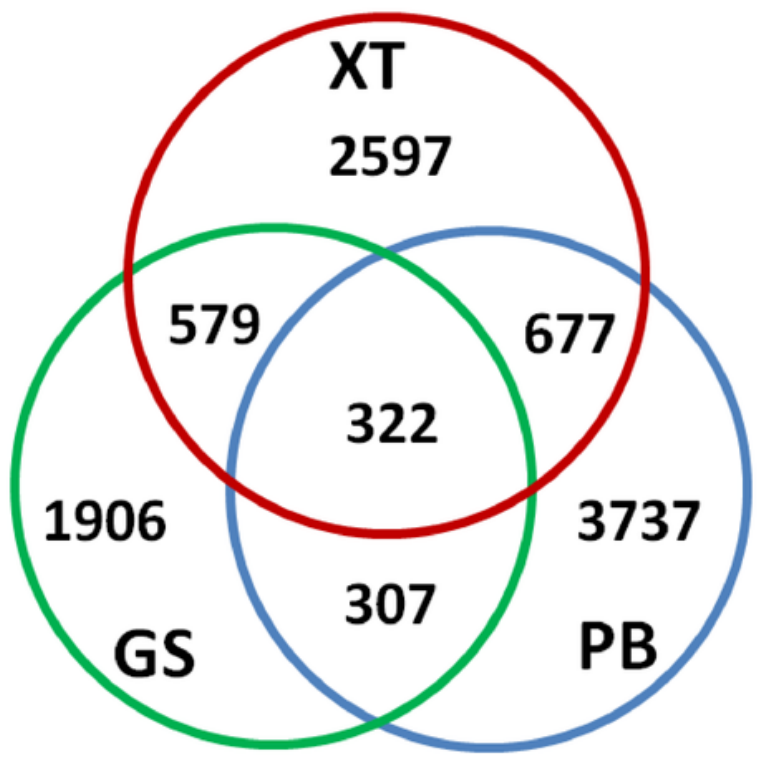

B Up-regulated genes in shoots

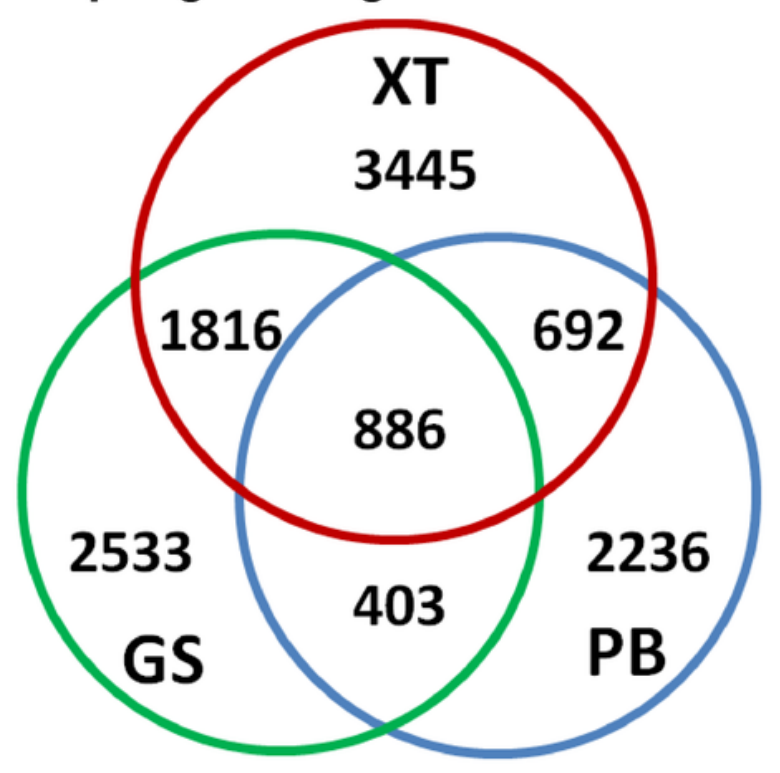


Figure 5

The expression levels of the genes encoding enzymes in terpenoid backbone and monoterpenoid biosynthesis pathways in P. Iactiflora

The heatmap plot presents the mean value of expression levels of the three biological replicates. The symbols are given according to their expression levels. $S$ and $R$ give shoots and roots respectively. 


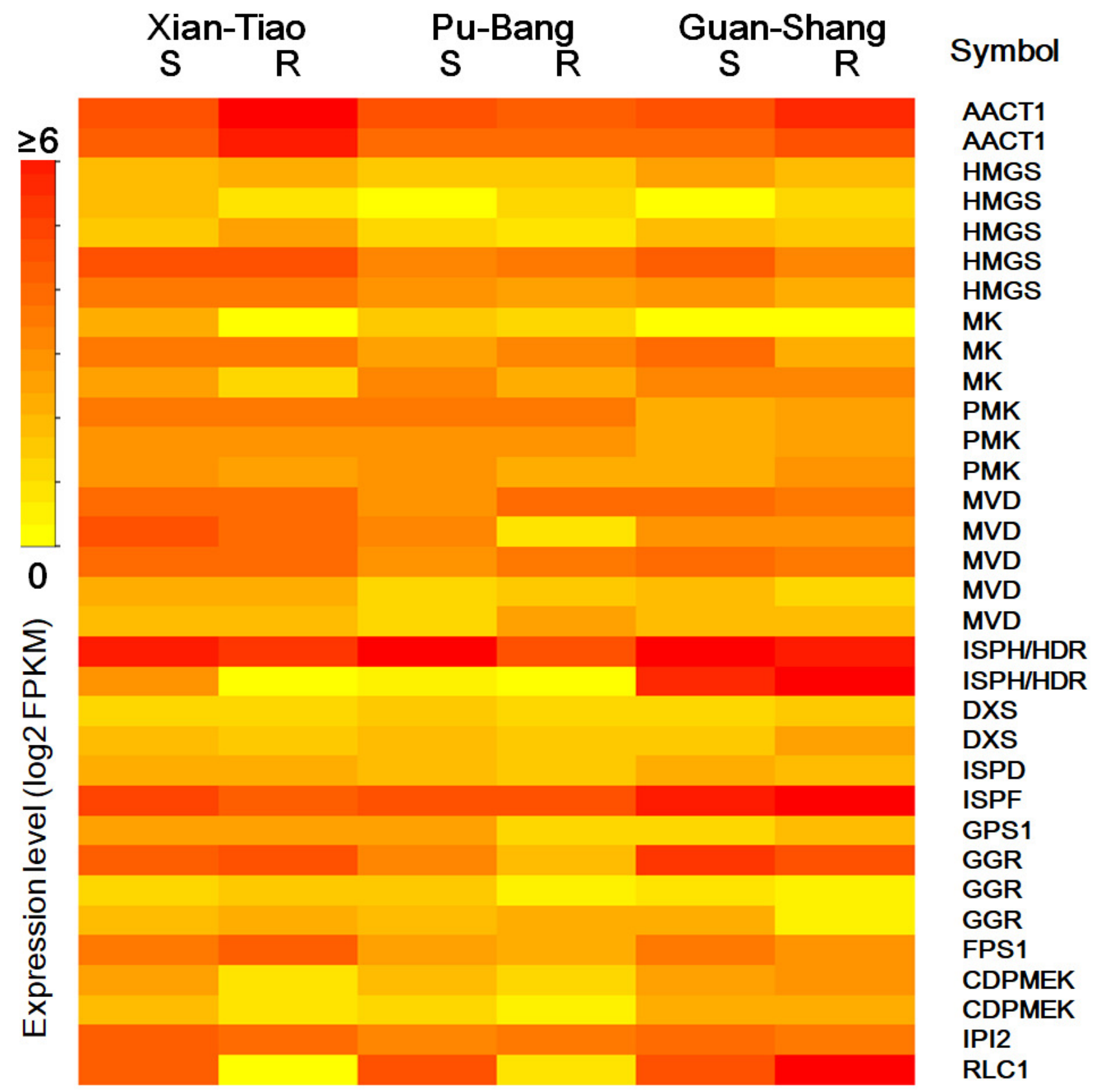


Figure 6

Expression levels of the genes verified by qRT-PCR

(A-J) show the relative expression levels of the unigenes encoding BHLH94, FPS1, FPS1, DREB1D, AACT1, AACT1, HMGS, IPI2, NAC098, MK, respectively. The expression levels of GS root samples were used for normalization of the relative expression levels. Bars give standard errors of biological replicates $(n=3)$. $S$ and $R$ give shoots and roots, respectively.

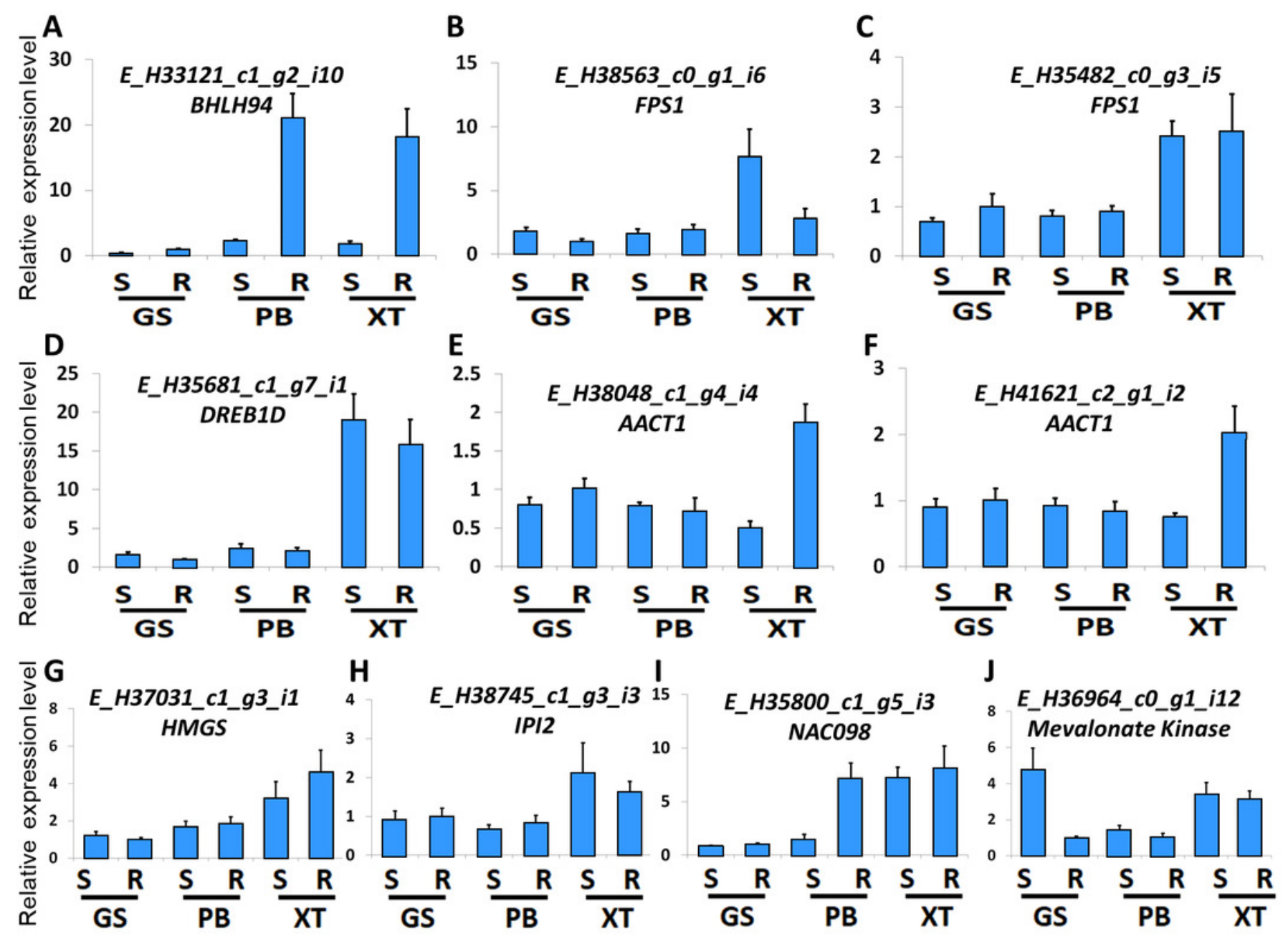




\section{Table $\mathbf{1}$ (on next page)}

Table 1. Transcriptome assembly of $P$. lactiflora unigenes 
Table 1. transcriptome assembly of P. lactiflora unigenes

\begin{tabular}{lr}
\hline Item & Value \\
\hline Total unigenes & 36264 \\
Total trinity transcripts & 72910 \\
GC content (\%) & 42.7 \\
Contig N10 (nt) & 1901 \\
Contig N20 (nt) & 1471 \\
Contig N30 (nt) & 1195 \\
Contig N40 (nt) & 986 \\
Contig N50 (nt) & 812 \\
Median contig length (nt) & 481 \\
Average contig length (nt) & 640.8 \\
Total assembled bases & 46721207 \\
\hline
\end{tabular}

1 